\title{
Virtual MIMO Beamforming and Device Pairing Enabled by Device-to-Device Communications for Multidevice Networks
}

\author{
Yeonjin Jeong, ${ }^{1}$ Jooheum Yoon, ${ }^{1}$ Sang Hyun Lee, ${ }^{2}$ and Yun Hee Kim ${ }^{1}$ \\ ${ }^{1}$ Department of Electronics and Radio Engineering, Kyung Hee University, Yongin, Gyeonggi-do 17104, Republic of Korea \\ ${ }^{2}$ School of Electrical Engineering, Korea University, Seoul 02841, Republic of Korea \\ Correspondence should be addressed to Yun Hee Kim; yheekim@khu.ac.kr
}

Received 23 November 2016; Accepted 30 January 2017; Published 21 May 2017

Academic Editor: Jeongyeup Paek

Copyright (C) 2017 Yeonjin Jeong et al. This is an open access article distributed under the Creative Commons Attribution License, which permits unrestricted use, distribution, and reproduction in any medium, provided the original work is properly cited.

\begin{abstract}
We consider a multidevice network with asymmetric antenna configurations which supports not only communications between an access point and devices but also device-to-device (D2D) communications for the Internet of things. For the network, we propose the transmit and receive beamforming with the channel state information (CSI) for virtual multiple-input multiple-output (MIMO) enabled by $\mathrm{D} 2 \mathrm{D}$ receive cooperation. We analyze the sum rate achieved by a device pair in the proposed method and identify the strategies to improve the sum rate of the device pair. We next present a distributed algorithm and its equivalent algorithm for device pairing to maximize the throughput of the multidevice network. Simulation results confirm the advantages of the transmit CSI and D2D cooperation as well as the validity of the distributive algorithm.
\end{abstract}

\section{Introduction}

The Internet of things (IoT) connecting billions of devices is anticipated to make our society smarter by collecting the remotely sensed data from the devices and actuating the devices autonomously based on the collected data $[1,2]$. The networks for the IoT would connect the devices not only to an access point (AP) but also to neighboring devices through direct communication links, which are often observed in machine-to-machine (M2M) and device-to-device (D2D) communications [3-5]. In addition, the direct links are controlled by the network as a whole or in part to guarantee the quality-of-services.

This paper utilizes the direct links of M2M and D2D communications to assist the information transfer from an AP to the devices through cooperation. There exist various cooperation methods such as amplify-and-forward (AF), decode-and-forward (DF), compress-and-forward (CF), and their variations to enlarge the coverage area or to improve the reliability of information delivery [6-9]. Among the methods, CF-based receive cooperation has attracted attention recently as a method of improving the system capacity by forming a virtual multiple-input multiple-output (MIMO) configuration with single-antenna devices [10-13].
The CF-based receive cooperation is considered for an AP with two antennas supporting two devices in $[10,11]$ from the aspect of physical layer designs with adaptive modulation and coding. A higher layer issue of device matching and resource sharing has been studied for the network with multiple devices in $[12,13]$, where a distributive message passing algorithm is derived based on the bargaining in exchange networks [14-18]. These studies have shown that the CFbased receive cooperation not only enjoys a proximity gain of the closely located devices but also provides a flexibility in the design of the D2D links by allowing any type of physical layer standard for the D2D links.

This paper proposes a network-assisted D2D communication network as one wireless communication platform for the IoT, which forms the virtual MIMO with multipleantenna devices to enhance the rate from the physical layer aspect and implements the distributed device pairing algorithms to improve the overall network throughput. The contributions of this paper are summarized as follows. Firstly, the beamforming $(\mathrm{BF})$ and power allocation $(\mathrm{PA})$ strategies exploiting the transmit CSI are proposed for the virtual MIMO to improve the rate performance of the downlink physical layer. Secondly, several distributive algorithms for device pairing are derived from the bargaining [15] and 
auction [19] to provide the network throughput equivalent to that of the optimal pairing. Thirdly, we verify the synergy of combining the virtual MIMO and device pairing algorithms under various system configurations in the scenario of IoT with D2D communications. It should be further noted that the D2D-assisted virtual MIMO with its enhancements can be adopted in the mobile cellular networks providing the proximity services [4] and the distributive pairing algorithms can be combined with various cooperation strategies among the devices for information sharing and processing [9].

The rest of this paper is organized as follows. After providing notations used in this paper, we describe the system model with and without D2D cooperation in Section 2. Section 3 investigates several methods to improve the sum rate of the two devices in D2D cooperation. Device pairing algorithms are devised from bargaining and auction to maximize the network throughput in Section 4. The performance of the network is evaluated in different aspects in Section 5 followed by the concluding remarks in Section 6 .

Notations. We denote by $\mathbf{A}^{T}, \mathbf{A}^{H}, \operatorname{tr}[\mathbf{A}]$, and $[\mathbf{A}]_{k, l}$ the transpose, Hermitian, trace, and $(k, l)$ th element of a matrix A, respectively; $\operatorname{diag}(\mathbf{x})$ denotes the diagonal matrix with diagonal vector $\mathbf{x}, \mathbf{I}_{N}$ denotes the $N \times N$ identity matrix, and $\mathbf{0}_{N \times M}$ denotes the $N \times M$ matrix with all-zero elements. We denote by $\mathbb{C}^{m \times n}$ the space for $m \times n$ complex matrices. The operator $E[\cdot]$ denotes the expectation, signifies "distributed as," and $\mathscr{C} \mathscr{N}(\overline{\mathbf{x}}, \boldsymbol{\Sigma})$ denotes the distribution of a vector $\mathbf{x}$ with complex Gaussian elements with mean $\overline{\mathbf{x}}$ and covariance matrix $\Sigma$.

\section{System Model}

Consider an IoT network with an AP and $K$ devices as described in Figure 1(a), where the AP is equipped with $N_{T}$ transmit antennas and each device is equipped with $N_{R}$ receive antennas. We consider asymmetric antenna configurations as $N_{T} \geq 2 N_{R}$, which are often encountered in practical scenarios with high complexity APs and low complexity IoT devices. The downlink (DL) from the AP to the devices utilizes a licensed spectrum of bandwidth $B_{C}$ for long-range communications, while the D2D links utilize an unlicensed spectrum of bandwidth $B_{D}$ for relatively shortrange communications as shown in Figure 1(b); although an unlicensed spectrum is also available at a very higher frequency as $60 \mathrm{GHz}$, we limit our description to an unlicensed spectrum at a carrier frequency below $6 \mathrm{GHz}$ which has a propagation model similar to that of the licensed spectrum. The DL frame is divided into $K$ equilength time slots, each of which is dedicated to each device to be shared with the paired device.

The channel from the AP to device $k$ is modeled by the channel response matrix $\mathbf{H}_{k}=\left[\mathbf{h}_{k, 1} \mathbf{h}_{k, 2}, \ldots, \mathbf{h}_{k, N_{R}}\right]^{T} \epsilon$ $\mathbb{C}^{N_{R} \times N_{T}}$. The component vector is modeled as $\mathbf{h}_{k, i} \sim$ $\mathscr{C} \mathscr{N}\left(\mathbf{0}_{N_{T} \times 1}, \omega_{k} \chi_{k} \mathbf{I}_{N_{T}}\right)$, where $\omega_{k}=\omega_{o} d_{k}^{-v}$ is the path-loss at device $k$ determined by the distance $d_{k}$ from the AP, path-loss constant $\omega_{o}$, and path-loss exponent $v$, and $\chi_{k}$ is the log-normal random variable reflecting the shadowing.

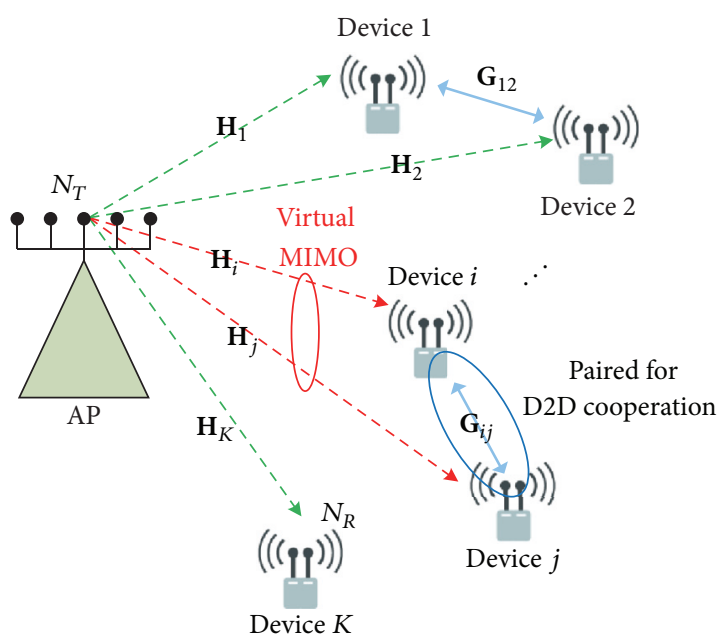

(a)

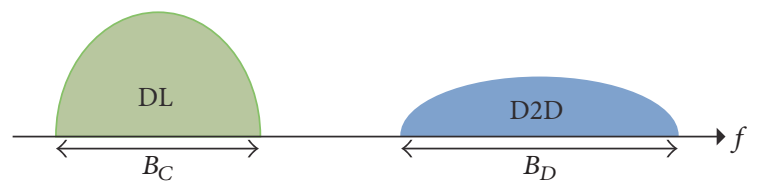

(b)

FIGURE 1: System model: (a) network configuration; (b) frequency bands.

The D2D link from device $i$ to device $j$ is described by the channel matrix $\mathbf{G}_{i j}=\left[\mathbf{g}_{i j, 1}, \mathbf{g}_{i j, 2}, \ldots, \mathbf{g}_{i j, N_{R}}\right]$, where $\mathbf{g}_{i j, k} \sim$ $\mathscr{C} \mathcal{N}\left(\mathbf{0}_{N_{R} \times 1}, \omega_{i j} \chi_{i j} \mathbf{I}_{N_{R}}\right)$ and $\omega_{i j}=\omega_{o} d_{i j}^{-v}$ is the path-loss between devices $i$ and $j$ at distance $d_{i j}$. The channel reciprocity holds in D2D links as $\mathbf{G}_{i j}=\mathbf{G}_{j i}$. This paper assumes that the AP has the perfect CSI on the channels $\left\{\mathbf{H}_{k}\right\}_{k=1}^{K}$. The network pairs the devices for signal transmission with transmit beamforming $(\mathrm{BF})$ over the time slots allocated to the devices. Without $\mathrm{D} 2 \mathrm{D}$ receive cooperation, the network supports $N_{T} \times N_{R}$ MIMO from the AP to each device in the pair. If the devices in the pair participate in $\mathrm{D} 2 \mathrm{D}$ receive cooperation, the network forms $N_{T} \times 2 N_{R}$ virtual MIMO from the AP and each device in the pair which can support $\min \left(N_{T}, 2 N_{R}\right)$ spatially multiplexed symbols.

Let devices $i$ and $j$ be paired for signal transmission over their time slots with transmit BF $\mathbf{W}_{i j}$. The transmit symbol vector for devices $(i, j)$ is modeled as

$$
\mathbf{x}_{i j}=\sqrt{\theta P_{A}} \mathbf{W}_{i j} \mathbf{s}_{i j}
$$

where $P_{A}$ is the transmit power of the AP, $\mathbf{s}_{i j}$ are the $2 N_{R} \times 1$ symbol vector with $E\left[\mathbf{s}_{i j} \mathbf{s}_{i j}^{H}\right]=\left(1 / 2 N_{R}\right) \mathbf{I}_{2 N_{R}}$, and $\theta=2 N_{R} /$ $\operatorname{tr}\left[\mathbf{W}_{i j} \mathbf{W}_{i j}^{H}\right]$ is the scaling factor satisfying the transmit power $P_{A}$. The corresponding received signal vector at device $k \in$ $\{i, j\}$ is expressed as

$$
\mathbf{y}_{k}=\left[y_{k, 1}, y_{k, 2}, \ldots, y_{k, N_{R}}\right]^{T}=\sqrt{\theta P_{A}} \mathbf{H}_{k} \mathbf{W}_{i j} \mathbf{s}_{i j}+\mathbf{n}_{k},
$$

where $\mathbf{n}_{k} \sim \mathscr{C} \mathscr{N}\left(\mathbf{0}_{N_{R} \times 1}, \sigma^{2} \mathbf{I}_{N_{R}}\right)$ is the background-noise vector at device $k$. 
With D2D cooperation that exchanges the compressed versions of the received signals between the paired devices, the received signals available at device $k \in\{i, j\}$ are given by

$$
\widetilde{\mathbf{y}}_{i j}=\left[\mathbf{y}_{i}^{T}, \widetilde{\mathbf{y}}_{j}^{T}\right]^{T}=\sqrt{\theta P_{A}} \mathbf{H}_{i j} \mathbf{W}_{i j} \mathbf{s}_{i j}+\widetilde{\mathbf{n}}_{i j}
$$

where $\mathbf{H}_{i j}=\left[\begin{array}{ll}\mathbf{H}_{i}^{T} & \mathbf{H}_{j}^{T}\end{array}\right]^{T}$ is the channel matrix of the pair and $\widetilde{\mathbf{n}}_{i j}=\left[\begin{array}{cc}\mathbf{n}_{i}^{T} & \widetilde{\mathbf{n}}_{j}^{T}\end{array}\right]^{T}$ with $\widetilde{\mathbf{n}}_{j}=\mathbf{n}_{j}+\mathbf{n}_{\mathrm{Q}, j}$. Here, $\mathbf{n}_{\mathrm{Q}, j}=\left[n_{\mathrm{Q}, j, 1}\right.$, $\left.n_{\mathrm{Q}, j, 2}, \ldots, n_{\mathrm{Q}, j, N_{R}}\right]^{T}$ is a vector of noises that the compression in $\mathrm{D} 2 \mathrm{D}$ cooperation incurs. With Voronoi vector quantization, the compression noises can be modeled as zero-mean complex Gaussian with $E\left[\mathbf{n}_{\mathrm{Q}, j} \mathbf{n}_{\mathrm{Q}, j}^{H}\right]=\operatorname{diag}\left(\boldsymbol{\sigma}_{\mathrm{Q}, j}^{2}\right)[10]$, where $\boldsymbol{\sigma}_{\mathrm{Q}, j}^{2}=\left[\sigma_{\mathrm{Q}, j, 1}^{2}, \sigma_{\mathrm{Q}, j, 2}^{2}, \ldots, \sigma_{\mathrm{Q}, j, N_{R}}^{2}\right]^{T}$ and $\sigma_{\mathrm{Q}, j, m}^{2}$ is bounded from the rate-distortion theory [20] as

$$
\begin{aligned}
\bar{\sigma}_{\mathrm{Q}, j, m}^{2} & =\frac{E\left[\left|y_{j, m}\right|^{2} \mathbf{h}_{j, m}\right]}{2^{r_{Q}, j}-1} \\
& =\frac{\sigma^{2}+\theta P_{A}\left|\mathbf{h}_{j, m}^{T} \mathbf{W}_{i j} \mathbf{W}_{i j}^{H} \mathbf{h}_{j, m}^{*}\right|}{2^{r_{Q} j}-1},
\end{aligned}
$$

where $r_{Q, j}$ is the source coding rate employed at device $j$ for compression of $\left\{y_{j, m}\right\}_{m=1}^{N_{R}}$. Therefore, we have

$$
\mathbf{R}_{\widetilde{\mathbf{n}}_{i j}}=E\left[\widetilde{\mathbf{n}}_{i j} \widetilde{\mathbf{n}}_{i j}^{H}\right]=\left[\begin{array}{cc}
\sigma^{2} \mathbf{I}_{N_{R}} & \mathbf{0}_{N_{R} \times N_{R}} \\
\mathbf{0}_{N_{R} \times N_{R}} & \operatorname{diag}\left(\boldsymbol{\sigma}_{Q, j}^{2}\right)
\end{array}\right] .
$$

Under this model, the network employs the transmit BF at the BS; that is,

$$
\mathbf{W}_{i j, \mathrm{~d} 2 \mathrm{~d}}=\widetilde{\mathbf{V}}_{i j}
$$

and $\theta_{\mathrm{d} 2 \mathrm{~d}}=1$, where $\widetilde{\mathbf{V}}_{i j} \in \mathbb{C}^{2 N_{T} \times 2 N_{R}}$ is the matrix constructed with the first $2 N_{R}$ columns of $\mathbf{V}_{i j}$ from singular value decomposition (SVD) of the pair channel matrix $\mathbf{H}_{i j}=$ $\mathbf{U}_{i j} \Sigma_{i j} \mathbf{V}_{i j}^{H}$. Device $i$ receiving signals in (3) through D2D cooperation uses the receive $\mathrm{BF} \mathbf{U}_{i j}^{H}$ such that

$$
\widetilde{\mathbf{z}}_{i j}=\mathbf{U}_{i j}^{H} \widetilde{\mathbf{y}}_{i j}=\sqrt{P_{A}} \Sigma_{i j} \mathbf{s}_{i j}+\mathbf{U}_{i j}^{H} \widetilde{\mathbf{n}}_{i j} .
$$

With D2D cooperation, the transmit symbol vector $\mathbf{s}_{i j}$ can be devoted to symbol transmission, for device $i$ only, for device $j$ only, or for both, according to the resource allocation.

For comparison, we consider the system without D2D cooperation. Up to $N_{R}$ symbols can be multiplexed for each device at the AP as $\mathbf{s}_{i j}=\left[\mathbf{s}_{i}^{T}, \mathbf{s}_{j}^{T}\right]^{T}$, where $\mathbf{s}_{k} \in \mathbb{C}^{N_{R} \times 1}$ is the symbol vector for device $k \in\{i, j\}$. The transmit BF $\mathbf{W}_{i j \text {,no }}$ without D2D cooperation can be designed as

$$
\mathbf{W}_{i j, \mathrm{no}}=\left[\mathbf{W}_{i, \mathrm{no}}, \mathbf{W}_{j, \mathrm{no}}\right] \text {, }
$$

by means of the block diagonalization (BD) method to decode $\mathbf{s}_{k}$ from (2) at device $k \in(i, j)$ as in [21]. To be specific, $\mathbf{W}_{i \text {,no }}$ is designed to be orthogonal to $\mathbf{H}_{j}$ but to be steered to $\mathbf{H}_{i}$ as $\mathbf{W}_{i, \text { no }}=\mathbf{V}_{j}^{\perp} \mathbf{V}_{i, \text { eq }}$, where $\mathbf{V}_{j}^{\perp} \in \mathbb{C}^{N_{T} \times\left(N_{T}-N_{R}\right)}$ is the submatrix of $\mathbf{V}_{j}$ corresponding to the null space of $\mathbf{H}_{j}=\mathbf{U}_{j} \boldsymbol{\Sigma}_{j} \mathbf{V}_{j}^{H}$ and $\widetilde{\mathbf{V}}_{i, \text { bd }} \in \mathbb{C}^{\left(N_{T}-N_{R}\right) \times N_{R}}$ is the submatrix corresponding to nonzero eigenvalues from the right unitary matrix $\mathbf{V}_{i, \mathrm{bd}}$ of $\mathbf{H}_{i} \mathbf{V}_{j}^{\perp}=\mathbf{U}_{i, \mathrm{bd}} \boldsymbol{\Sigma}_{i, \mathrm{bd}} \mathbf{V}_{i, \mathrm{bd}}^{H}$. The design of $\mathbf{W}_{j, \text { no }}$ is obtained in a similar way. Therefore, we have

$$
\mathbf{W}_{i j, \mathrm{no}}=\left[\mathbf{V}_{j}^{\perp} \widetilde{\mathbf{V}}_{i, \mathrm{bd}}, \mathbf{V}_{i}^{\perp} \widetilde{\mathbf{V}}_{j, \mathrm{bd}}\right]
$$

and $\theta_{\text {no }}=1$. By applying $\mathbf{U}_{k \text {,bd }}^{H}$ to $\mathbf{y}_{k}$ at device $k \in\{i, j\}$, we have

$$
\mathbf{z}_{k}=\mathbf{U}_{k, \mathrm{bd}}^{H} \mathbf{y}_{k}=\sqrt{P_{A}} \boldsymbol{\Sigma}_{k, \mathrm{bd}} \mathbf{s}_{k}+\mathbf{U}_{k, \mathrm{bd}}^{H} \mathbf{n}_{k} .
$$

It should be noted that $2 N_{R}$ received signals are available at each device, while $N_{R}$ received signals are available at each device, and thus BF strategies are different.

\section{Improvement of Sum Rate of a Device Pair}

With D2D cooperation, the sum rate achieved by the pair $(i, j)$ is a linear combination of rates $\widetilde{\mathscr{R}}_{i j}$, delivered to device $i$ when the two time slots are devoted to device $i$, and $\widetilde{\mathscr{R}}_{j i}$, delivered to device $j$ when the two time slots are devoted to device $j$. The sum rate achieved by a device pair is given by

$$
\mathcal{S}_{i j}=\kappa_{i} \widetilde{\mathscr{R}}_{i j}+\kappa_{j} \widetilde{\mathscr{R}}_{j i} \text { bps, }
$$

where nonnegative factors $\kappa_{i}$ and $\kappa_{j}$ denote the fractions of the two time slots allocated to devices $i$ and $j$, respectively, subject to $\kappa_{i}+\kappa_{j}=1$. Here, $\widetilde{\mathscr{R}}_{i j}$ is given by

$$
\widetilde{\mathscr{R}}_{i j}=\frac{2 B_{C}}{K} \sum_{m=1}^{2 N_{R}} \log _{2}\left(1+\frac{P_{A}}{2 N_{R} \sigma_{j, m}^{2}} \lambda_{i j, m}\right) \text { bps }
$$

from (7), where $\lambda_{i j, m}=\left[\Sigma_{i j}^{2}\right]_{m, m}$ and

$$
\sigma_{j, m}^{2}=\left[\mathbf{U}_{i j}^{H} \mathbf{R}_{\widetilde{\mathbf{n}}_{i, j}} \mathbf{U}_{i j}\right]_{m, m} .
$$

The rate $\widetilde{\mathscr{R}}_{j i}$ delivered to device $j$ is also obtained in a similar form by exchanging $j$ and $i$.

To improve the rate (12), we need to reduce $\sigma_{j, m}^{2}$ or equivalently $\bar{\sigma}_{\mathrm{Q}, j, m}^{2}$ given in (4). We can reduce $\bar{\sigma}_{\mathrm{Q}, j, m}^{2}$ by increasing the source coding rate $r_{Q, j}$ as much as possible. The source coding rate for device $j$ to compress the received signal samples $\left\{y_{j, m}\right\}_{m=1}^{N_{R}}$ to be forwarded to device $i$ is limited by the channel capacity

$$
C_{i j}=B_{D} \log _{2} \operatorname{det}\left(\mathbf{I}_{N_{R}}+\frac{P_{D}}{N_{R} \sigma_{D}^{2}} \mathbf{G}_{i j} \mathbf{G}_{i j}^{H}\right)
$$


of the D2D link, where $P_{D}$ and $\sigma_{D}^{2}$ are the transmit power and noise power of the device, respectively. Specifically, for the forwarded samples to be recovered at device $i$ without errors, their transmission rate $B_{C} N_{R} r_{Q, j}$ over the D2D link should not exceed $C_{i j}$. Therefore, the source coding rate $r_{\mathrm{Q}, j}$ is upperbounded by

$$
r_{\mathrm{Q}, i j}^{\max }=\frac{\eta}{N_{R}} \log _{2} \operatorname{det}\left(\mathbf{I}_{N_{R}}+\frac{P_{D}}{N_{R} \sigma_{D}^{2}} \mathbf{G}_{i j} \mathbf{G}_{i j}^{H}\right),
$$

where $\eta=B_{D} / B_{C}$ is the bandwidth ratio.

With the maximum source coding rate, the variance of the compression noise is given by

$$
\bar{\sigma}_{\mathrm{Q}, j, m}^{2} \leq \frac{\sigma^{2}\left(1+\left(P_{A} / 2 N_{R} \sigma^{2}\right)\left\|\mathbf{h}_{j, m}\right\|^{2}\right)}{\operatorname{det}\left(\mathbf{I}_{N_{R}}+\left(P_{D} / N_{R} \sigma_{D}^{2}\right) \mathbf{G}_{i j} \mathbf{G}_{i j}^{H}\right)^{\eta / N_{R}}-1}
$$

from (4) and (15). From (16), we can suggest some strategies to improve the sum rate achieved by a device pair in D2D cooperation. Firstly, the compression nose variance decreases exponentially as the bandwidth expansion $\eta$ increases. For the fixed bandwidth expansion ratio as $\eta=1$, the compression noise variance is reduced when the D2D link quality is much better than the DL quality in an average sense as

$$
\frac{P_{A}}{\sigma^{2}} \omega_{j} \ll \frac{P_{D}}{\sigma_{D}^{2}} \omega_{i j}
$$

We further improve the sum rate of the device pair by applying power allocation (PA) $\psi_{i j}=\left[\psi_{i i, 1}, \psi_{i i, 2}, \ldots, \psi_{i i, 2 N_{R}}\right]^{T}$ such that $E\left[\mathbf{s}_{i j} \mathbf{s}_{i j}^{H}\right]=\operatorname{diag}\left(\psi_{i j}\right)$ and $\sum_{m=1}^{2 N_{R}} \psi_{i j, m}=1$. In the case, the sum rate of the device pair is given by

$$
\widetilde{\mathscr{R}}_{i j}=\frac{2 B_{C}}{K} \sum_{m=1}^{2 N_{R}} \log _{2}\left(1+\frac{P_{A}}{\sigma_{j, m}^{2}} \lambda_{i j, m} \psi_{i j, m}\right) \mathrm{bps},
$$

which is maximized by applying the water-filing algorithm with the information on $\left\{\left(P_{A} / \sigma_{j, m}^{2}\right) \lambda_{i j, m}\right\}_{m=1}^{2 N_{R}}$ [20]. Although the CSI on $\left\{\lambda_{i j, m}\right\}_{m=1}^{M}$ is available at the AP, the noise variance $\sigma_{j, m}^{2}$ is not easy to be informed to the AP since it depends on the transmit signal and D2D link qualities. Therefore, the AP can compute the PA with water-filling algorithm by assuming $\sigma_{j, m}^{2}=\sigma^{2}$. This sum rate of the device pair becomes the maximum when $\sigma_{j, m}^{2}=\sigma^{2}$ or equivalently when $\eta=\infty$.

For comparison, the achievable sum rate of pair $(i, j)$ using two slots without D2D cooperation is given by $S_{i j \text {, no }}=$ $R_{i, \text { no }}+R_{j, \text { no }}$, where

$$
R_{k, \text { no }}=\frac{2 B_{C}}{K} \sum_{m=1}^{N_{R}} \log _{2} \operatorname{det}\left(1+\frac{P_{A}}{2 N_{R} \sigma^{2}} \lambda_{k, m, \text { bd }}\right) \text { bps }
$$

from (10), where $\lambda_{k, m, \mathrm{bd}}=\left[\Sigma_{k, b d}^{2}\right]_{m, m}$.

\section{Distributed Pairing Algorithms}

This section aims at forming the pairs to maximize the average normalized throughput of the network. The problem is formulated as

$$
\begin{array}{ll}
\max _{\Pi} & T_{\mathrm{avg}}=\frac{1}{B_{C}} \sum_{i=1}^{K} \sum_{j=i+1}^{K} E\left\{\mathcal{S}_{i j} \pi_{i j}\right\} \mathrm{bps} / \mathrm{Hz}, \\
\text { s.t. } & \sum_{i=1}^{K} \pi_{i j}=1, \\
& \sum_{j=1}^{K} \pi_{i j}=1, \\
& \pi_{i j}=\pi_{j i}, \\
& \pi_{i j} \in\{0,1\}, \\
\forall i, j,
\end{array}
$$

where $\Pi$ is the $K \times K$ matrix with $[\Pi]_{i, j}=\pi_{i j}$. The pairing indicator $\pi_{i j} \in\{0,1\}$ is given by $\pi_{i j}=1$ when devices $i$ and $j$ are paired and $\pi_{i j}=0$, otherwise.

We first solve the problem by using a distributed algorithm based on Nash bargaining as proposed in [12] not only to maximize the average throughput but also to satisfy each device's desire to obtain the best bargaining gain. We then derive the auction algorithms for device paring which are equivalent to the bargaining algorithm.

4.1. Bargaining Algorithm Based on Message Passing. The problem is described by a graph $(\mathscr{V}, \mathscr{E})$ in an exchange network [14], where $\mathscr{V}$ is the set of vertices corresponding to the devices and $\mathscr{E}$ is the set of edges corresponding to the D2D links among the devices. The edge $(i, j) \in \mathscr{E}$ connecting vertices $i$ and $j$ is associated with a positive weight

$$
w_{i j}=\delta_{i j}^{\min }=\min \left(\widetilde{\mathscr{R}}_{i j}, \widetilde{\mathscr{R}}_{j i}\right)
$$

which corresponds to the sum rate of the device pair $(i, j)$ ensured by any sharing policy $\left(\kappa_{i}, \kappa_{j}\right)$; without knowing $\left(\kappa_{i}, \kappa_{j}\right)$ in advance, we estimate the sum rate of the device pair pessimistically with the minimum value.

Let $(\mathscr{M}(\boldsymbol{\Pi}), \zeta)$ denote a configuration of the device pairing and resource sharing, where $\mathscr{M}(\Pi)$ is a matching described by $\Pi$ in graph $(\mathscr{V}, \mathscr{E})$ and $\zeta=\left\{\zeta_{i}: i \in \mathscr{V}\right\}$ is the set of the rates that the devices can obtain from the matching $\mathscr{M}(\boldsymbol{\Pi})$. The solution is obtained with a balanced configuration [14] satisfying the stability

$$
\zeta_{i}+\zeta_{j}=w_{i j}
$$

for device pair $(i, j)$ contained in the matching $\mathscr{M}(\boldsymbol{\Pi})$ and the balance

$$
\zeta_{i}-\max _{k \in \mathcal{N}(i) \backslash j}\left(w_{i k}-\zeta_{k}\right)_{+}=\zeta_{j}-\max _{l \in \mathcal{N}(j) \backslash i}\left(w_{j l}-\zeta_{l}\right)_{+}
$$

for all $(i, j) \in \mathscr{M}(\Pi)$, where $\mathcal{N}(i)$ is the set of neighbors of device $i$. 


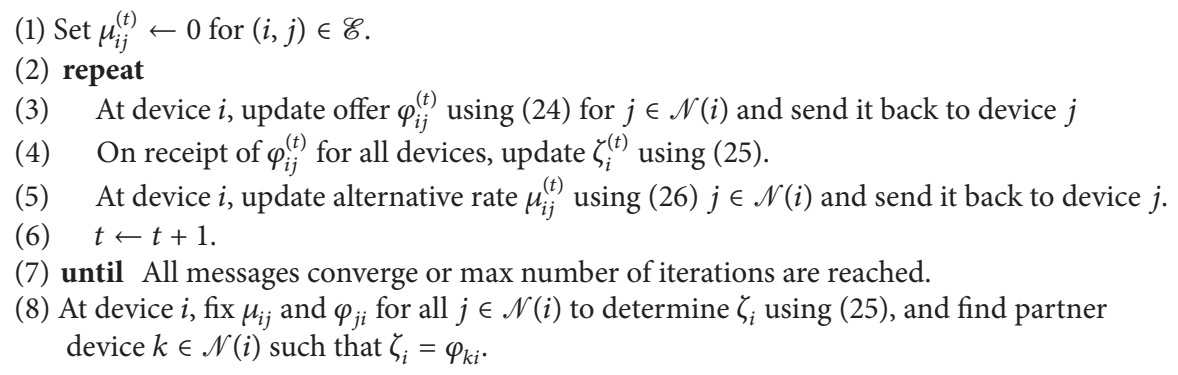

Algorithm 1: Distributed matching and resource sharing algorithm.

This stable balanced configuration can be found by a distributed algorithm based on a message passing (MP) framework [17, 18, 22, 23], where each device transfers a nonnegative message on the current best alternative in device matching to its neighbors at each time instant. Let $\mu_{i j}^{(t)}$ denote the message from device $i$ to device $j$ at time $t$, representing the resource share which is guaranteed for device $i$ in the pair $(i, j)$. That is, $\mu_{i j}^{(t)}$ is an alternative rate that device $i$ can obtain maximally up to time instant $t$ when pairing with its neighbor $k \in \mathcal{N}(i) \backslash j$ except for $j$. Initially, $\mu_{i j}^{(0)}$ is set to zero for all $(i, j) \in \mathscr{E}$ since there is no guarantee ensured by the neighboring devices initially. Based on the alternative rate, an individual device makes an offer $\varphi_{i j}^{(t)}$ to each of its neighbors for $\mathrm{D} 2 \mathrm{D}$ cooperation, where the offer from device $i$ to device $j$ is computed as

$$
\varphi_{i j}^{(t)}=\left(w_{i j}-\mu_{i j}^{(t)}\right)_{+}-\frac{1}{2}\left(w_{i j}-\mu_{i j}^{(t)}-\mu_{j i}^{(t)}\right)_{+} .
$$

With the offers from the other devices, device $i$ estimates its maximal rate using

$$
\zeta_{i}^{(t)}=\max _{k \in \mathcal{N}(i)} \varphi_{k i}^{(t)}
$$

The update rule for $\mu_{i j}^{(t)}$ is given by

$$
\begin{aligned}
& \mu_{i j}^{(t+1)}=(1-\xi) \mu_{i j}^{(t)}+\xi \tilde{\mu}_{i j}^{(t+1)}, \\
& \tilde{\mu}_{i j}^{(t+1)}=\max _{k \in \mathcal{N}(i) \backslash j} \varphi_{k i}^{(t)},
\end{aligned}
$$

where $\tilde{\mu}_{i j}^{(t+1)}$ is an estimate of the rate guaranteed for device $i$ and $\xi \approx 0.7$ is the damping factor [12]. With the updated $\mu_{i j}^{(t+1)}$, the repeated updates of $\varphi_{i j}^{(t+1)}$ and $\zeta_{i}^{(t+1)}$ proceed using (24) and (25), respectively. The iteration continues until $\mu_{i j}^{(t+1)}$ converges for all available pairs $(i, j)$. Let $\mu_{i j}, \varphi_{i j}$, and $\zeta_{i}$ denote the corresponding values to which $\mu_{i j}^{(t)}, \varphi_{i j}^{(t)}$, and $\zeta_{i}^{(t)}$ converge, that is, a fixed point of the algorithm. Upon convergence, device $i$ sets its share to $\zeta_{i}$ and chooses device $k \in \mathcal{N}(i)$ with $\varphi_{k i}=\zeta_{i}$ as its partner. From $\zeta_{i}$, the device obtains the resource sharing factor $\kappa_{i}=\zeta_{i} / w_{i j}$. This procedure is summarized in Algorithm 1.
4.2. Equivalent Auction Algorithm for Device Pairing. The auction algorithm finds the maximum weighted matching (MWM) via an auction, based on the notion of buyers and prices [19]. The MWM corresponds to the optimal pairing providing the maximum throughput so that we briefly describe the auction algorithm in terms of messages defined in the previous section.

We can set the alternative rate $\mu_{i j}^{(t)}$ to the price which buyer $i$ pays to buy product $j$ and set the sum rate $w_{i j}$ to the value of product $j$, respectively. The benefit that can be earned when buyer $i$ buys product $j$ is given by $w_{i j}-\mu_{i j}^{(t)}$. The goal is to find the assignment of all buyers and products that maximizes the overall benefit, that is, $\sum_{(i, j)}\left(w_{i j}-\mu_{i j}^{(t)}\right) \pi_{i j}$. Therefore, the sum $\sum_{(i, j)} \mu_{i j}^{(t)} \pi_{i j}$ of the prices is constant and the MWM maximizes the overall benefit [15]. To handle this problem, the auction algorithm proceeds as in Algorithm 2. This algorithm is known to converge to the MWM if a solution exists $[15,19]$.

In this algorithm, the maximum is taken over all incoming $\varphi_{i j_{i}}^{(t)}$ when updating $\mu_{i j}^{(t+1)}$. In addition, device $i$ does not bid at every iteration and to every neighboring device, thereby slowing down the convergence speed. Also, the auction algorithm works only for a bipartite network where one class of buyers is assigned only to the other class of products. Thus, the algorithm should be modified to allow the MWM for nonbipartite network where buyers can also become products. Note that, in a nonbipartite network, the net benefit of device $i$ corresponds to the price that device $j$ should pay to form a pair $(i, j)$. Subsequently, device $i$ does not necessarily consider the offer from device $j$ to determine the price that device $j$ should pay for pairing. To all of those objectives, the auction algorithm is modified to obtain a new algorithm running in a nonbipartite network, and the resulting algorithm is summarized in Algorithm 3.

In this algorithm, device $i$ can bid to all neighboring device $j$ at each iteration instead of updating $\varphi_{i j}^{(t)}$ by adding a small constant $\delta$. Here, note that the auction algorithm in this subsection bears very close resemblance to the bargaining algorithm in Section 4.1. If we drop the second term in (24), which corresponds to dividing the surplus rate gain equally, we end up with Algorithm 3. Thus, the developed algorithm only differs from the modified auction algorithm in that it 
(1) Set $\mu_{i j}^{(t)} \leftarrow 0$ for $(i, j) \in \mathscr{E}$.

(2) repeat

(3) At device $i$, calculate

$$
\begin{aligned}
& v_{i}=\max _{j}\left(w_{i j}-\mu_{i j}^{(t)}\right)_{+}, \\
& j_{i}=\underset{j}{\operatorname{argmax}}\left(w_{i j}-\mu_{i j}^{(t)}\right)_{+}, \\
& u_{i}=\max _{k \neq j_{i}}\left(w_{i k}-\mu_{i k}^{(t)}\right)_{+},
\end{aligned}
$$

and update offer $\varphi_{i j}^{(t)}$ using $\varphi_{i j}^{(t)}=w_{i j_{i}}-u_{i}+\delta$ with a certain positive constant $\delta$ and send it to device $j_{i}$.

(4) On receipt of $\varphi_{i j_{i}}^{(t)}$ at each device $j_{i}$, calculate alternative rate gain $\mu_{i j}^{(t)}$ using (26) and send it back to device $i$.

(5) $\quad t \leftarrow t+1$.

(6) until All messages converge or max number of iterations are reached.

(7) At device $i$, fix $\mu_{i j}$ and $\varphi_{j i}$ for all $j \in \mathcal{N}(i)$ to determine $\zeta_{i}$ using (25), and find partner device $k \in \mathcal{N}(i)$ such that $\zeta_{i}=\varphi_{k i}$.

Algorithm 2: Auction algorithm.

(1) Set $\mu_{i j}^{(t)} \leftarrow 0$ for $(i, j) \in \mathscr{E}$.

(2) repeat

(3) At device $i$, update offer $\varphi_{i j}^{(t)}$ for $j \in \mathcal{N}(i)$ using $\varphi_{i j}^{(t)}=\left(w_{i j}-\mu_{i j}^{(t)}\right)_{+}$and send it back to device $j$.

(4) On receipt of $\varphi_{i j}^{(t)}$ for all devices, update $\zeta_{i}^{(t)}$ using (25).

(5) At device $i$, update alternative rate gain $\mu_{i j}^{(t)}$ using (26) for $j \in \mathscr{N}(i)$ and send it back to device $j$.

(6) $t \leftarrow t+1$.

(7) until All messages converge or max number of iterations are reached.

(8) At device $i$, fix $\mu_{i j}$ and $\varphi_{j i}$ for all $j \in \mathcal{N}(i)$ to determine $\zeta_{i}$ using (25), and find partner device $k \in \mathcal{N}(i)$ such that $\zeta_{i}=\varphi_{k i}$.

Algorithm 3: Modified auction algorithm.

includes an extra term that reduces "offers" by half the surplus rate gain. In fact, this algorithm is proved to converge to the MWM if a unique solution exists using the same technique as [12].

We now consider the generalization of the message update rule for (24) as

$$
\varphi_{i j}^{(t)}=\left(w_{i j}-\mu_{i j}^{(t)}\right)_{+}-\gamma\left(w_{i j}-\mu_{i j}^{(t)}-\mu_{j i}^{(t)}\right)_{+} .
$$

We see that (27) leads to the bargaining algorithm in Section 4.1 with $\gamma=1 / 2$ and the modified auction algorithm with $\gamma=0$, respectively. In fact, for $\gamma \in[0,1)$, the corresponding algorithm finds the MWM if a unique solution exists. This statement can be proved using the technique in [16]. Thus, the distributive algorithms provided in this work can also find the MWM.

\section{Numerical Results}

We evaluate the performance of the network with $K=8$ devices when $P_{A}=29 \mathrm{dBm}, P_{D}=23 \mathrm{dBm}, B_{C}=10 \mathrm{MHz}$, and $\sigma^{2}=\sigma_{D}^{2}=-104.5 \mathrm{dBm}$. The devices are clustered in the circle of radius $r_{o}$ of which the center is located at distance $d_{o}$ from the AP. In the circle, the location of the devices is randomly generated with uniform distribution. The SNR at the center of the cluster circle is denoted by $\rho_{o}$ for a reference parameter. We assume the unlicensed spectrum of bandwidth $B_{D}=10 \mathrm{MHz}, 20 \mathrm{MHz}$, and $40 \mathrm{MHz}$ for $\eta=1, \eta=2$, and $\eta=4$. The path-loss exponent is set to $v=3.76$ and the shadowing deviation is set to $10 \mathrm{~dB}$ for both the licensed and unlicensed spectrums.

Figure 2 shows the performance of the cluster with $d_{o}=$ $300 \mathrm{~m}$ and $r_{o}=200 \mathrm{~m}$ as the cluster center SNR $\rho_{o}$ varies when $\eta=2, N_{T}=2, N_{R}=1$, and $\omega_{o}=\rho_{o}-133.5 \mathrm{in} \mathrm{dB}$. In the figure, "D2D-PA" and "D2D" indicate the proposed schemes with water-filling PA and equal PA, respectively, while "No $\mathrm{D} 2 \mathrm{D}$ " denotes the BD-based BF without D2D cooperation and "D2D (No CSI)" indicates the D2D cooperation without the transmit CSI; here, "D2D (No CSI)" applies the zeroforcing receive $\mathrm{BF}$ at the devices. For the benchmark, we also provide the performance with ideal D2D link ("Ideal") having no compression noise, or equivalently $\eta=\infty$. The lines in the figure correspond to the results with optimal device matching obtained by finding the maximum value after searching for all possible pairing configurations while the marks correspond to the results with the distributive algorithm presented in the paper. The figure shows that the distributive algorithm provides the performance almost indistinguishable from the optimal performance. The transmit CSI on the DL improves the average throughput for both cases of the network with D2D cooperation and with no D2D cooperation. The best performance is achieved with "D2D-PA" exploiting the transmit CSI and D2D cooperation.

Figure 3 illustrates how the pairs are matched by the distributive bargaining algorithm for a single channel realization generated in Figure 2 when $\rho_{o}=10 \mathrm{~dB}$. The left and right 


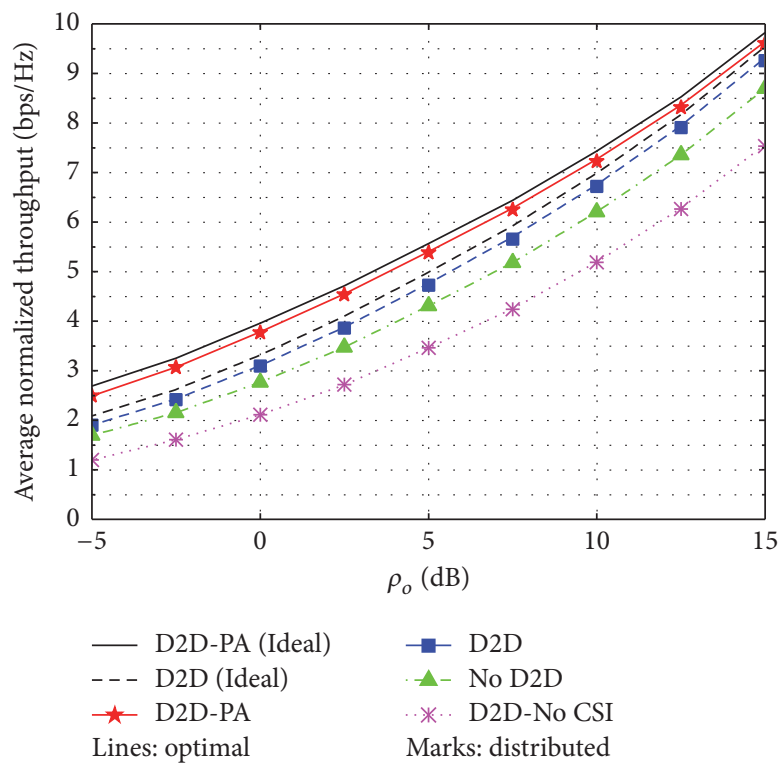

FIGURE 2: Average normalized throughput of the cluster of radius $r_{o}=200 \mathrm{~m}$ located at $d_{o}=300 \mathrm{~m}$ as a function of the cluster center SNR $\rho_{o}$ when $\eta=1, N_{T}=4$, and $N_{R}=2$.

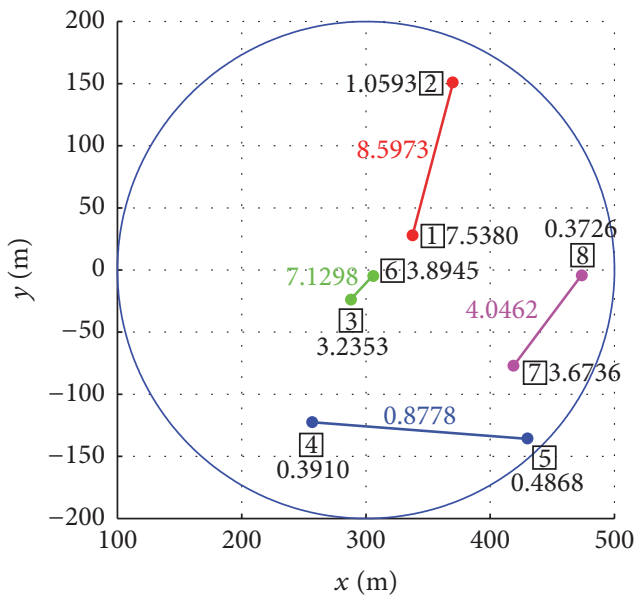

(a)

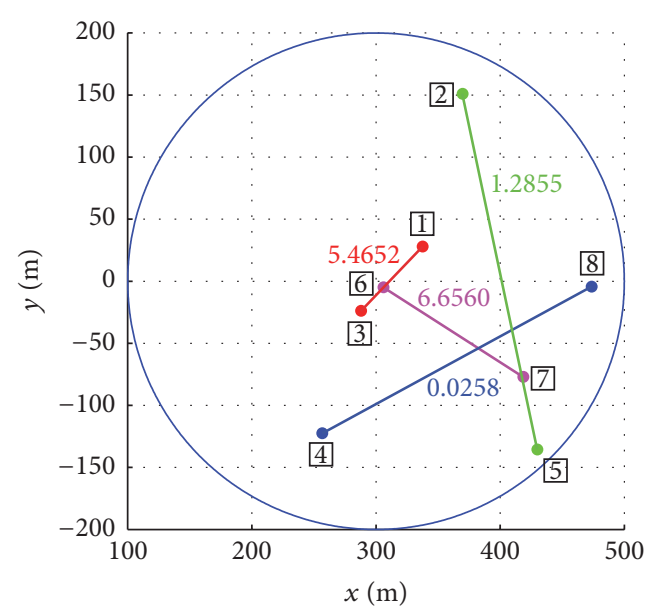

(b)

FIgURE 3: A single realization of the device pairing and resource sharing when $\rho_{o}=10 \mathrm{~dB}$ in Figure 2: (a) D2D; (b) No D2D.

subfigures describe a matching of "D2D" and "No D2D," respectively, for the same channel realization. The numbers inside the squares identify the devices and the numbers near to edges of the same color are the sum rates (weights) of the device pairs associated with the edges. We also show the rates (shares) of the devices near to the devices in Figure 3(a). Note that "D2D" can support the rates of the devices by choosing the resource sharing factors $\left(\kappa_{i}, \kappa_{j}\right)$ while "No D2D" cannot support them. With D2D cooperation, the devices in proximity are likely to be paired to improve the quality of the D2D links as shown in Figure 3(a). On the other hand, the devices without D2D cooperation tend to be paired independent of their locations but dependent on the DL channel qualities as shown in Figure 3(b). As expected from the distributive algorithm, a device in a better condition tends to take higher rate than the other device in the pair with $\mathrm{D} 2 \mathrm{D}$ cooperation; for instance, device 1 having more neighbors to bargain with tends to take higher rate than device 2 having less neighbors.

Figure 4 compares the performance of the network with that of transmit CSI for different antenna configurations $N_{T} \times N_{R}$ when $N_{T}=2 N_{R}$ and the other conditions remain unchanged from Figure 2. As the number $N_{T}\left(N_{R}\right)$ of antennas increases, the throughput is improved for all methods by multiplexing more symbols at the transmitter. In addition, the gain of "D2D-PA" over "No D2D" increases with the number of antennas by concentrating the signal power to the desired channel modes. Unfortunately, the performance gain with D2D cooperation is marginal for $2 \times 1$ antenna configuration. 


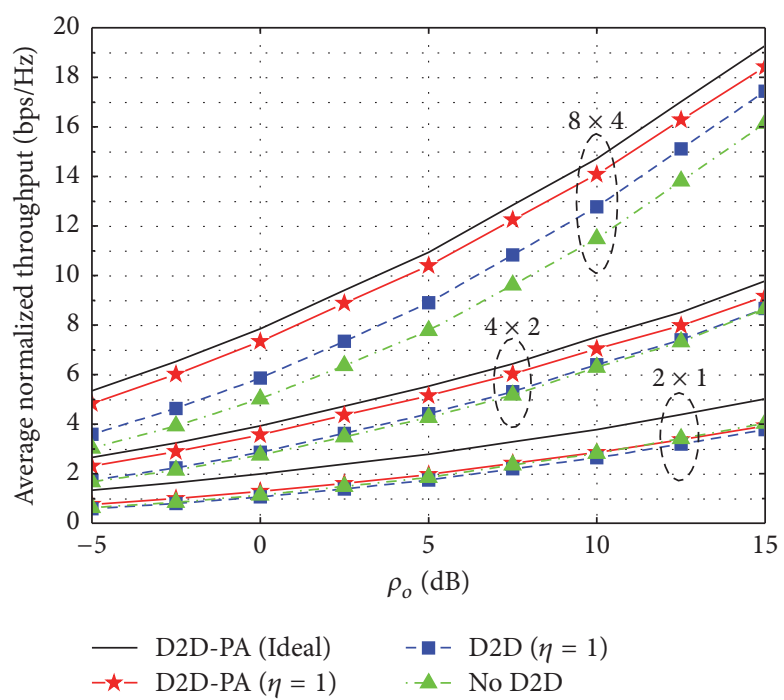

FIGURE 4: Average normalized throughput of the cluster for several antenna configurations as the cluster center $\operatorname{SNR} \rho_{o}$ varies.

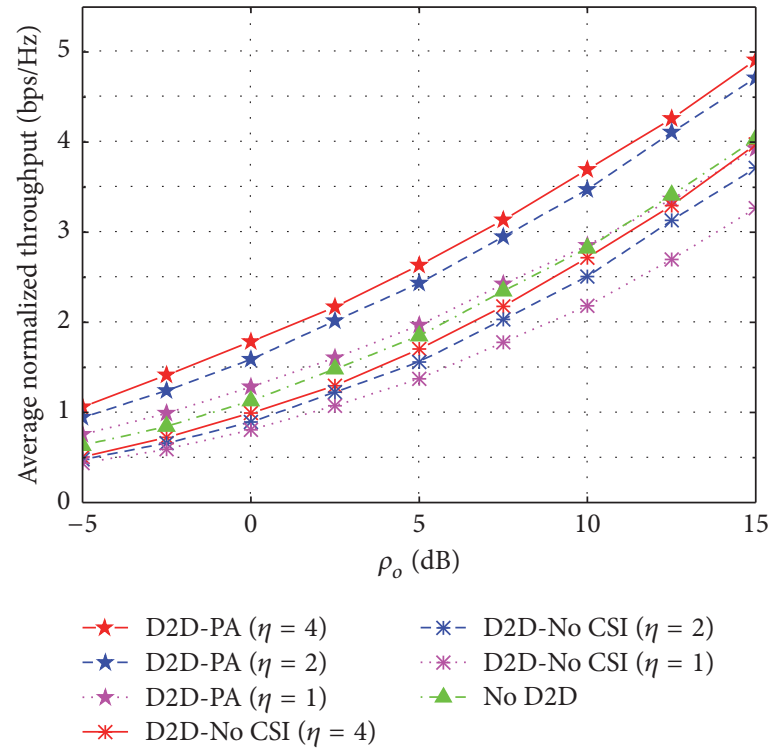

FIGURE 5: Average normalized throughput of the cluster as a function of the cluster center SNR $\rho_{o}$ for several $\eta$ values when $N_{T}=2$ and $N_{R}=1$.

To improve the performance of D2D cooperation for $N_{T}=2$ and $N_{R}=1$, we increase the bandwidth expansion ratio $\eta$ in Figure 5 while the other conditions remain unchanged from Figure 2. The D2D cooperation methods are benefited by improving the D2D link qualities through increasing the D2D bandwidth irrespective of the transmit CSI available at the AP or not. The gain is more prominent when $\eta$ increases from 1 to 2 .

Figure 6 compares the performances as the cluster location $d_{o}$ varies when $\eta=1$ and $r_{o}=25 \mathrm{~m}$. The antenna configuration is set to $N_{T}=2$ and $N_{R}=1$ in Figure 6(a) and $N_{T}=4$ and $N_{R}=2$ in Figure 6(b). The cluster center SNR is $0 \mathrm{~dB}$ at $d_{o}=d_{o, \max }=300 \mathrm{~m}$ for both figures and the cluster center SNR at distance $d_{o}$ is obtained from $\rho_{o}=\left(d_{o \text {, max }} / d_{o}\right)^{\nu}$.
The results reveal that the gain of $\mathrm{D} 2 \mathrm{D}$ cooperation is larger when the cluster location gets farther from the AP since the quality of the D2D links relative to the DL gets better. This observation comes from the fact that the signals with larger amplitude suffer from larger quantization errors for the fixed number of quantization levels. The figure also shows that the effect of the compression noises becomes negligible when the device cluster is as small as that used in the figure.

\section{Concluding Remarks}

This paper considers an IoT network supporting both the centralized signal transmission and D2D communications. With asymmetric antenna configurations, we propose the 

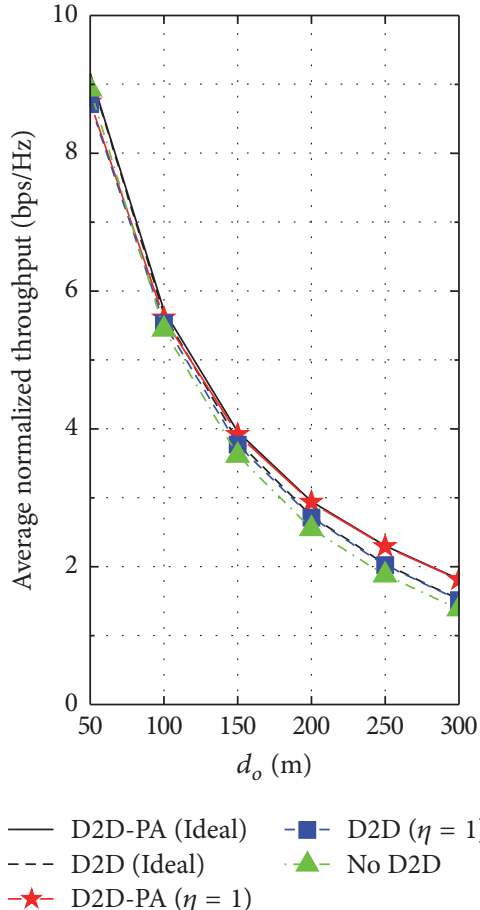

(a)

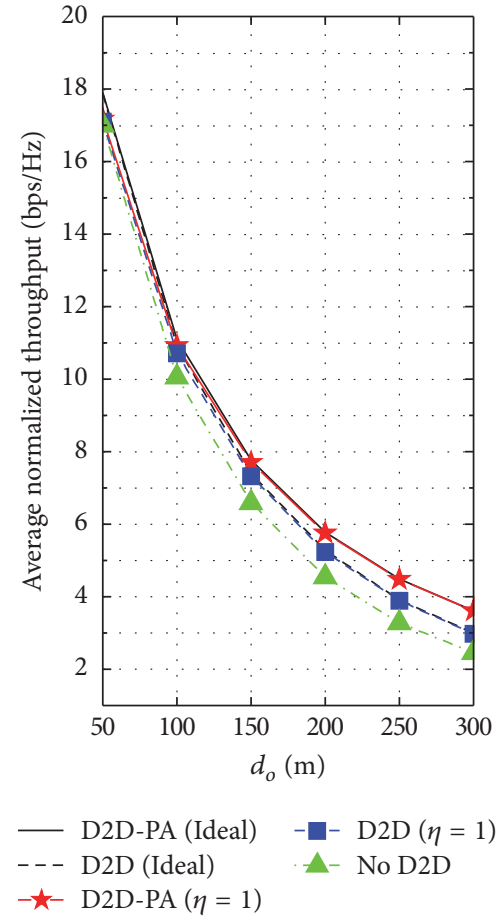

(b)

Figure 6: Average normalized throughput of the cluster as the cluster location $d_{o}$ varies when $\eta=1$ and $r_{o}=25 \mathrm{~m}$ : (a) $N_{T}=2, N_{R}=1$; (b) $N_{T}=4, N_{R}=2$.

transmit and receive BF for the virtual MIMO formed by D2D cooperation. For the proposed method, we provide the strategies to improve the sum rate of the device pair and a distributive algorithm for device pairing and resource sharing to maximize the network throughput while meeting each device's desire. It is confirmed that the distributed algorithm works well in the network with transmit CSI also, providing a performance close to that obtained with optimal pairing. The transmit CSI improves the performance of the network significantly even without D2D cooperation but the $\mathrm{D} 2 \mathrm{D}$ cooperation leverages the performance further. The performance gain of the $\mathrm{D} 2 \mathrm{D}$ cooperation becomes more prominent when the $\mathrm{D} 2 \mathrm{D}$ links are relatively better than the downlink by using a larger bandwidth for the D2D links and by applying the method to clusters of closely located devices but farther from the AP.

\section{Competing Interests}

The authors declare that they have no competing interests.

\section{Acknowledgments}

This work was supported by the National Research Foundation of Korea (NRF) grant funded by the Korea government (MSIP) (nos. NRF-2015R1A2A2A01005390 and NRF2015R1C1A1A01052529).

\section{References}

[1] A. Zanella, N. Bui, A. Castellani, L. Vangelista, and M. Zorzi, "Internet of things for smart cities," IEEE Internet of Things Journal, vol. 1, no. 1, pp. 22-32, 2014.

[2] J. Lee, K. D. Singh, Y. Hadjadj-Aoul, and N. Kumar, "Wireless and mobile technologies for the internet of things," Mobile Information Systems, vol. 2016, Article ID 8206548, 2 pages, 2016.

[3] M. Hasan, E. Hossain, and D. Niyato, "Random access for machine-to-machine communication in LTE-advanced networks: issues and approaches," IEEE Communications Magazine, vol. 51, no. 6, pp. 86-93, 2013.

[4] X. Lin, J. G. Andrews, A. Ghosh, and R. Ratasuk, "An overview of 3GPP device-to-device proximity services," IEEE Communications Magazine, vol. 52, no. 4, pp. 40-48, 2014.

[5] M. Tehrani, M. Uysal, and H. Yanikomeroglu, "Device-todevice communication in $5 \mathrm{G}$ cellular networks: challenges, solutions, and future directions," IEEE Communications Magazine, vol. 52, no. 5, pp. 86-92, 2014.

[6] J. N. Laneman, D. N. C. Tse, and G. W. Wornell, "Cooperative diversity in wireless networks: efficient protocols and outage behavior," Institute of Electrical and Electronics Engineers. Transactions on Information Theory, vol. 50, no. 12, pp. 3062-3080, 2004

[7] C. T. Ng, N. Jindal, A. J. Goldsmith, and U. Mitra, "Capacity gain from two-transmitter and two-receiver cooperation," IEEE Transactions on Information Theory, vol. 53, no. 10, pp. 38223827, 2007. 
[8] J. S. Wang, Y. H. Kim, I. Song, P. C. Cosman, and L. B. Milstein, "Cooperative relaying of superposition coding with simple feedback for layered source transmission," IEEE Transactions on Communications, vol. 61, no. 11, pp. 4448-4461, 2013.

[9] P. Li and S. Guo, Cooperative Device-to-Device Communication in Cognitive Radio Cellular Networks, Springer, Berlin, Germany, 2014.

[10] J. Jiang, J. S. Thompson, and H. Sun, "A singular-valuebased adaptive modulation and cooperation scheme for virtualMIMO systems," IEEE Transactions on Vehicular Technology, vol. 60, no. 6, pp. 2495-2504, 2011.

[11] J. Jiang, J. S. Thompson, H. Sun, and P. M. Grant, "Performance assessment of virtual multiple-input multiple-output systems with compress-and-forward cooperation," IET Communications, vol. 6, no. 11, pp. 1456-1465, 2012.

[12] S. H. Lee, D. R. Shin, H. W. Jeong, and Y. H. Kim, "Distributed bargaining strategy for downlink virtual MIMO with deviceto-device communication," IEEE Transactions on Communications, vol. 64, no. 4, pp. 1503-1516, 2016.

[13] Y. Jeong, J. Yoon, Y. H. Kim, and S. H. Lee, "Distributed matching and resource sharing for space division multiplexing assisted by D2D cooperation," in Proceedings of the International Conference on Information and Communication Technology Convergence (ICTC '16), pp. 112-114, Jeju-do, Korea, October 2016.

[14] J. Kleinberg and E. Tardos, "Balanced outcomes in social exchange networks," in Proceedings of the Fortieth Annual ACM Symposium on Theory of Computing (STOC '08), pp. 295-304, ACM, New York, NY, USA, 2008.

[15] M. Bayati, D. Shah, and M. Sharma, "Max-product for maximum weight matching: convergence, correctness, and LP duality," IEEE Transactions on Information Theory, vol. 54, no. 3, pp. 1241-1251, 2008.

[16] Y. Kanoria, M. Bayati, C. Borgs, J. Chayes, and A. Montanari, "Fast convergence of natural bargaining dynamics in exchange networks," in Proceedings of the 27th Annual ACM-SIAM Symposium on Discrete Algorithms, pp. 1518-1537, San Francisco, Calif, USA, January 2011.

[17] M. Shamaiah, S. H. Lee, S. Vishwanath, and H. Vikalo, "Distributed algorithms for spectrum access in cognitive radio relay networks," IEEE Journal on Selected Areas in Communications, vol. 30, no. 10, pp. 1947-1957, 2012.

[18] S. H. Lee, M. Shamaiah, H. Vikalo, and S. Vishwanath, "Message-passing algorithms for coordinated spectrum sensing in cognitive radio networks," IEEE Communications Letters, vol. 17, no. 4, pp. 812-815, 2013.

[19] D. P. Bertsekas, "Auction algorithms for network flow problems: a tutorial introduction," Computational Optimization and Applications, vol. 1, no. 1, pp. 7-66, 1992.

[20] T. M. Cover and J. A. Thomas, Elements of Information Theory, John Wiley \& Sons, Hoboken, NJ, USA, 2nd edition, 2006.

[21] Q. H. Spencer, A. L. Swindlehurst, and M. Haardt, "Zero-forcing methods for downlink spatial multiplexing in multiuser MIMO channels," IEEE Transactions on Signal Processing, vol. 52, no. 2, pp. 461-471, 2004.

[22] F. R. Kschischang, B. J. Frey, and H.-A. Loeliger, "Factor graphs and the sum-product algorithm," IEEE Transactions on Information Theory, vol. 47, no. 2, pp. 498-519, 2001.

[23] I. Sohn, S. H. Lee, and J. G. Andrews, "Belief propagation for distributed downlink beamforming in cooperative MIMO cellular networks," IEEE Transactions on Wireless Communications, vol. 10, no. 12, pp. 4140-4149, 2011. 

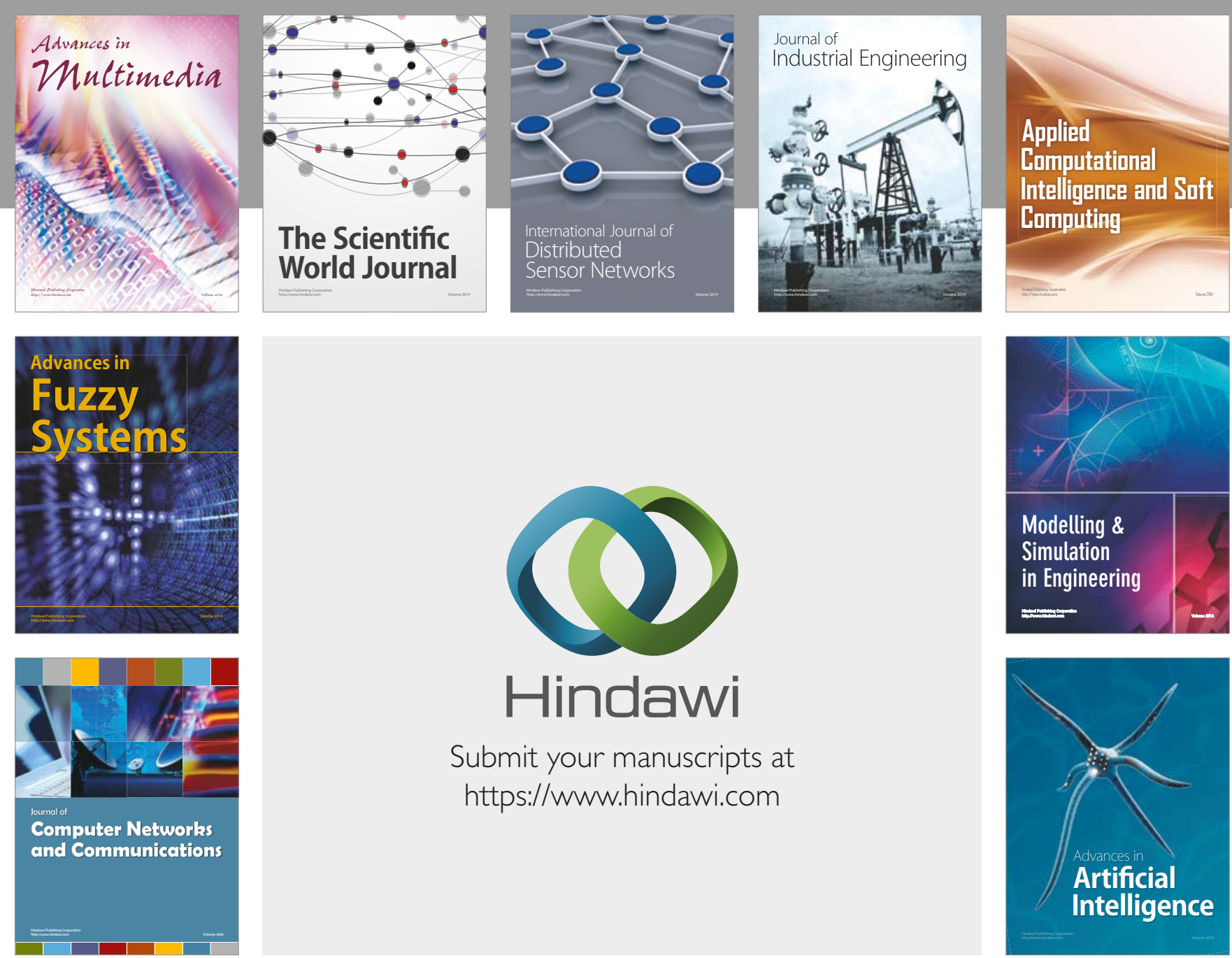

\section{Hindawi}

Submit your manuscripts at

https://www.hindawi.com
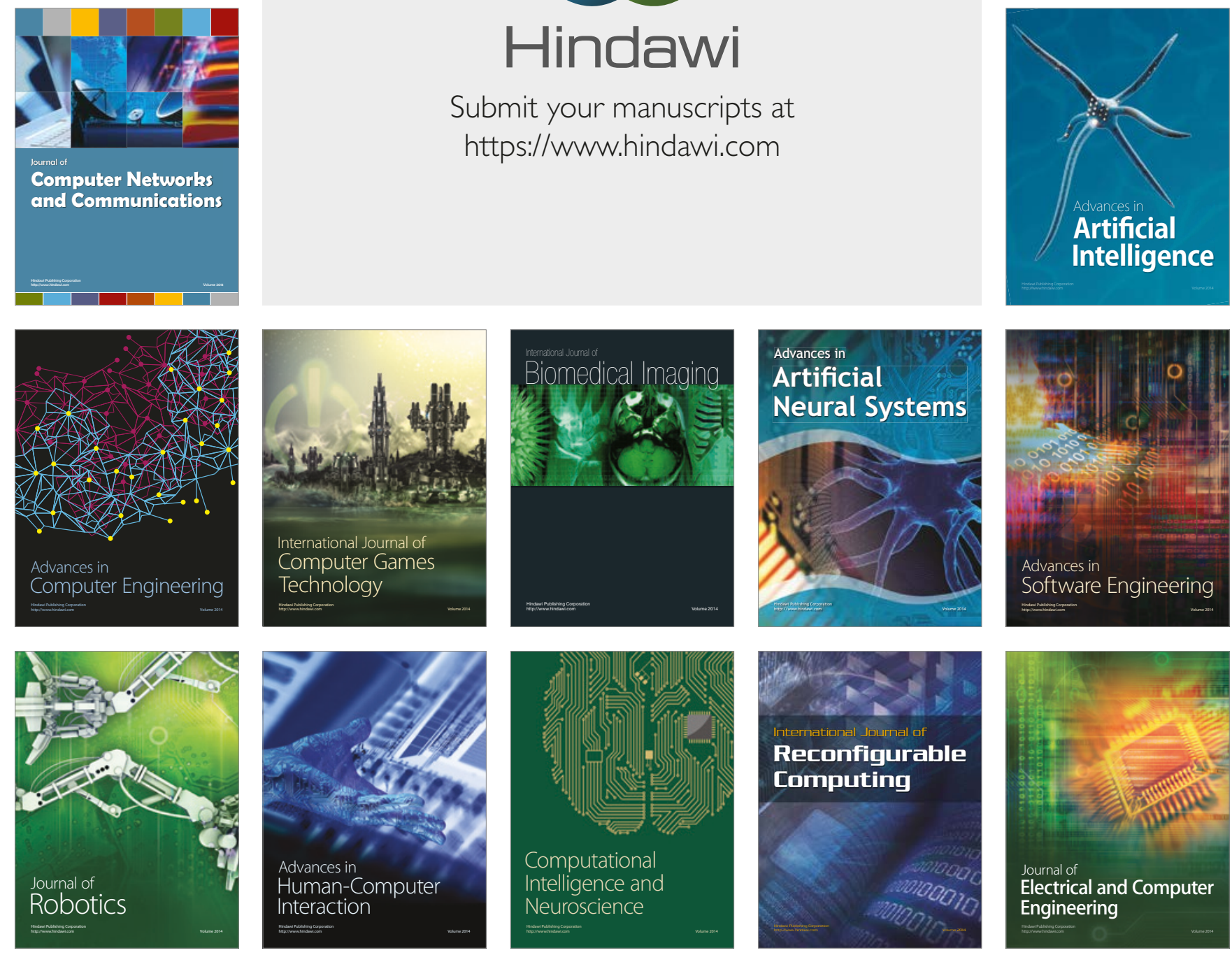